\title{
Designing and Optimization of Wheel Assembly of a Formula Student
}

\section{Car}

\author{
Joijode Vrushabh Umesh ${ }^{+*}$ and Yadav Abhishek ${ }^{\dagger}$ \\ †Mechanical Engineering Department, Vishwakarma Institute of Technology, 666, Upper Indira Nagar, Pune-37, India
}

Accepted 02 March 2016, Available online 15 March 2016, Special Issue-4 (March 2016)

\begin{abstract}
In a Formula Student Car or in any car as such, the part that connects the main frame of the body with the wheels through suspension arms is known as the Wheel Assembly. It is a part of the Final drive as well. While designing and developing any automobile the designing of the wheel assembly is critical. It is due to the reason that a lot of forces are acting on the wheel assembly during accelerating, braking, cornering and tilting. Furthermore, the Wheel Assembly is an important part of an automobile and its failure is hazardous endangering human life. Therefore is required to design the Wheel Assembly and its components considering all the factors leading to the failure by developing a safe Design. It must also be noted that, the components must be designed in such a way that they have a minimum weight at the same time care must be taken that they do not cross a certain limit of stress value. In this Paper the Complete Design Procedure of the Wheel Assembly for R12 Rims with wet Tires (165×60) has been presented along with optimization of the same components. The weight of the Vehicle is considered to be $300 \mathrm{~kg}$ along with the driver. The paper illustrates the forces acting on the components, the failure criteria and the optimization of the components. Optimization has been carried out by doing analysis of the components in Hyper mesh. The paper deals with finding out the dimensions of the individual components and also detecting the probable regions of stress concentration. The Wheel Assembly designed in the paper is of Team Veloce, VIT, Pune. The design procedure follows all the rules laid down by FSAE Rule Book for Formula Type Cars.
\end{abstract}

Keywords: Yield Strength, Factor of safety, Load transfer, stress concentration, mesh, element size, analysis.

\section{Introduction}

An automobile is said to function appropriately only when all its systems are working as they are required to work. The engine produces power and gives it to the drive train through a clutch. The portion of the drive which transmits this power from the drive train to the wheel and which connects the main frame of the body with the wheels through the suspension arms is known as the Wheel Assembly. It is a part of the final drive. Thus it serves the function of transmitting the power from the drive shaft to the wheels. Besides as the suspension arms are also connected to it, the wheel assembly also transfers the bump force from the ground to the suspension arms.

There are always two types of masses in an automobile - sprung and unsprung mass. All the mass of the vehicle that is damped by the spring is called as the sprung mass. As the Wheel Assembly mass is not damped by the spring, it comes into the unsprung mass category. We know that the unsprung mass must be lower than the sprung mass and also should be as

*Corresponding author: Joijode Vrushabh Umesh least as possible to provide proper drive stability and load balancing of the vehicle. Thus it becomes important to reduce the mass of the wheel assembly and the rims and tires. But while doing this care must be taken that the mass of the wheels, tires and the wheel assembly must be enough to prevent the lateral toppling of the vehicle at the time of cornering or impact.

There are a lot of forces acting on the wheels in the static and especially in the dynamic condition. As the Wheel assembly is directly connected to the wheels, all these forces also have an impact on the designing of the Wheel Assembly. A lot of forces act on the wheel assembly during accelerating, braking, cornering and tilting.

A good Wheel Assembly is one which can sustain such forces over a longer period of time. Thus it is required to design the wheel assembly considering all these factors. A failure of any component of the Wheel Assembly means a breakdown of the automobile and in some cases might also be hazardous for the driver. Thus utmost care must be taken while designing the Wheel Assembly. The objective of Optimization is 
always to find the best possible and suitable dimension. This is because optimization does not always mean reducing dimensions it also means finding out the dimensions which will just enough to sustain the forces.

Here in this case, R12 rims have been used along with $165 \times 60$ wet tires. The Weight of the vehicle is considered to be $300 \mathrm{~kg}$ along with the driver. All the forces have been found out on the above basis and according to the above mentioned Wheels. The Wheel Assembly designed in the paper is of Team Veloce, VIT, Pune. The design procedure follows all the rules laid down by FSAE Rule Book for Formula Type Cars.

The analysis is done on Hyper works.

While generating the mesh proximity and curvature feature is used which is used to generate more amount of nodes at bends and cavities.

The above feature is helpful as stress concentration tends to accumulate more in regions of bends and cavities.

Table 1 Parameters of mesh elements

\begin{tabular}{|c|c|}
\hline Parameter & Size \\
\hline Minimum Element Size & $0.4 \mathrm{~mm}$ \\
\hline Feature Angle & 30 \\
\hline Element Size & $2 \mathrm{~mm}$ \\
\hline
\end{tabular}

The mesh generated is a tetra mesh.

Table 2 Element type

\begin{tabular}{|c|c|}
\hline 2D Type & Trias \\
\hline 3D Type & Tetras \\
\hline
\end{tabular}

Elements generated after meshing are checked under the following parameters:

Table 3 Checking Parameters

\begin{tabular}{|c|c|}
\hline Aspect & $>5.00$ \\
\hline Warpage & $>5.00$ \\
\hline Skew & $>60.00$ \\
\hline Tet. Collapse & $<0.2$ \\
\hline Cell Squish & $>0.5$ \\
\hline Jacobian & $<0.7$ \\
\hline
\end{tabular}

$99 \%$ and above of all the elements generated in the meshing pass the following parameter.

\section{Components of Wheel Assembly.}

As shown in the figure the Wheel Assembly consists of the following Components.
1) Spindle-(1)
2) Knuckle-(1)
3) Hub-(1)
4) Bearings

a. Taper Roller Bearing- Front-(2)

b. Deep Groove Ball Bearing-Rear-(1)

6) Cotter/Split Pin-(1)

Before understanding the procedure it is important to understand how all these components are assembled. Firstly the spindle is take. The Knuckle is press fitted on the spindle. Then the spacer between the knuckle and hub is either slide fitted of=r press fitted on the spindle in order to accommodate the caliper. After wards the inner race of the bearing is press fitted on the spindle. The hub is taken and the outer race of the bearing is press fitted in the hub. Then the hub is positioned on the bearing and then the second bearing's inner race is press fitted on the spindle. Then the nut is tightened on the spindle and a split pin is inserted into the hole made in spindle for positively locking the Wheel Assembly.

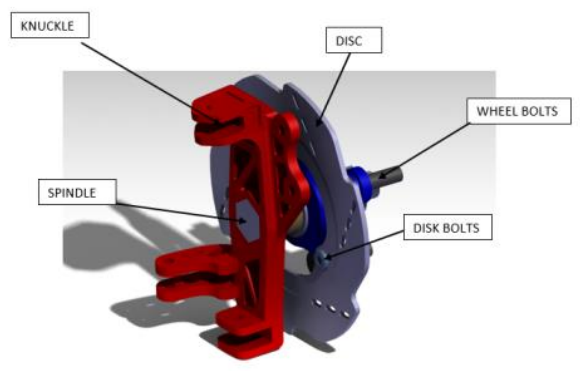

Fig.1 Wheel Assembly View-1

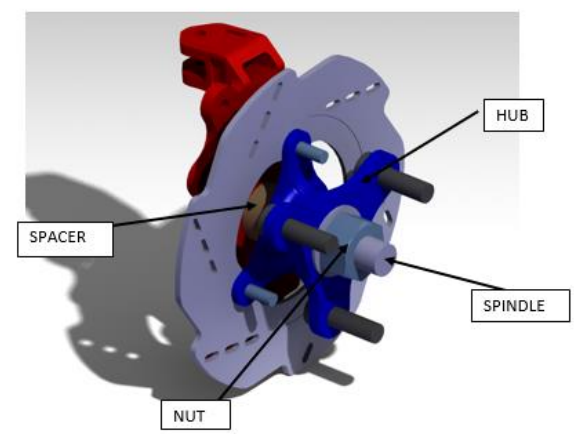

Fig.2 Wheel Assembly View-2 


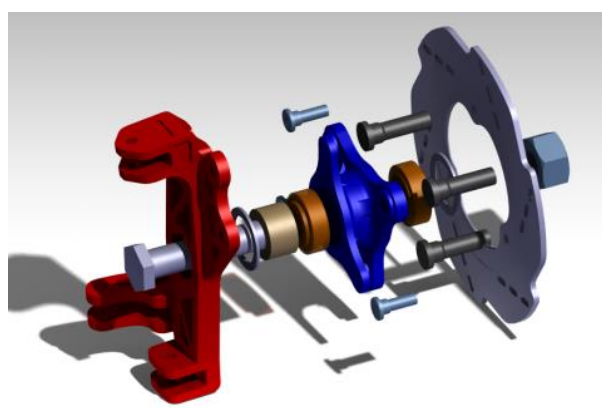

Fig.3 Exploded View

\section{Design Procedure}

The following paper deals with the design procedure of the wheel assembly. Designing the wheel assembly is nothing but deciding the shape according to the requirement, finding out the dimensions of the various components, their position in the wheel assembly, the type of fit between the components, etc. The following are the steps in designing the Wheel assembly:-

\subsection{Getting the prerequisite parameters from the suspension and steering geometry}

The first step while designing the wheel assembly is to find out the required parameters in order to design the wheel assembly from the steering and the suspension geometry. The Steering and Suspension Engineer design their geometry, a kinematic representation of various parts in that system, according to the requirement. A Wheel Assembly Design Engineer must refer to these geometries so that in the actual car these parameter are followed. The Steering Geometry has an influence on the Front Wheel Assembly only. The Front Suspension and Rear Suspension Geometry affect the front and rear assemblies. Parameters such as King Pin Angle, Steering Arm angle, Tie rod angle are obtained from the Steering geometry, whereas the Caster angle, the angle of upper A-arm and the lower A-arm, Rear Track width are obtained from Suspension Geometry. Parameters like the Stub length and the front track width are obtained from both Geometries. Considering the Front Wheel Assembly the Parameters are as follows

King Pine Inclination:-70

Caster Angle: - $5^{0}$

Tie Rod Angle: - $3.94^{0}$

Track Width: - 1200mm

Wheel Diameter: - 502.8mm

Total length of Knuckle: - 171mm

Upper A-arm angle: $-6.369^{\circ}$

Lower A-arm angle: - $3.193^{0}$

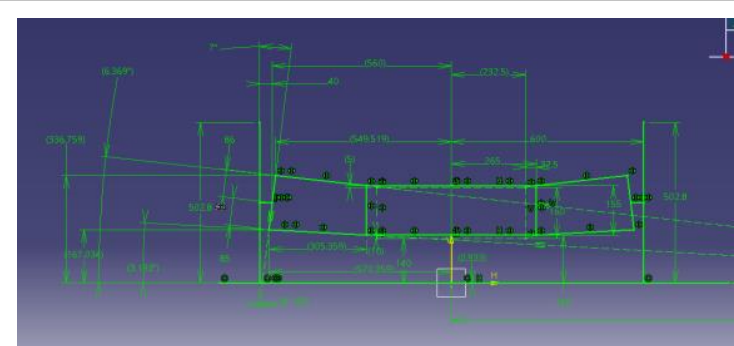

Fig.4 Front Suspension Geometry

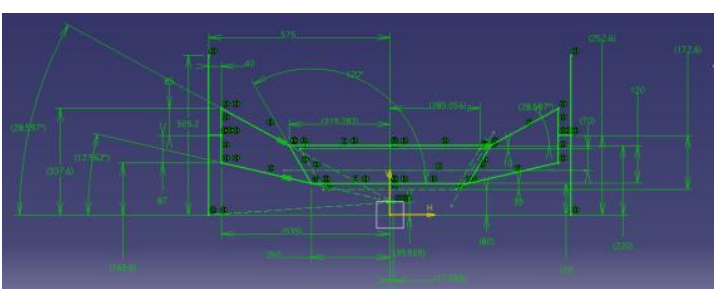

Fig.5 Rear Suspension Geometry

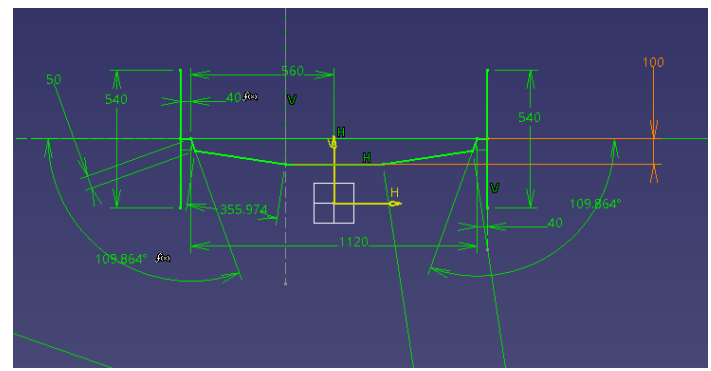

Fig.6 Steering Geometry

\subsection{Finding the effect length of knuckle}

The lengths of the knuckle obtained from the suspension geometry is from one view only. Besides it is a matter whether or not to provide inbuilt caster also affect the length. So it is important to find out the actual lengths from these two angles as shown in figure. From the above figure it is clear that the effective length of the front knuckle is 85.685 above and 86.761 from the center point.

\section{Total Effect Length: - 172.411mm}

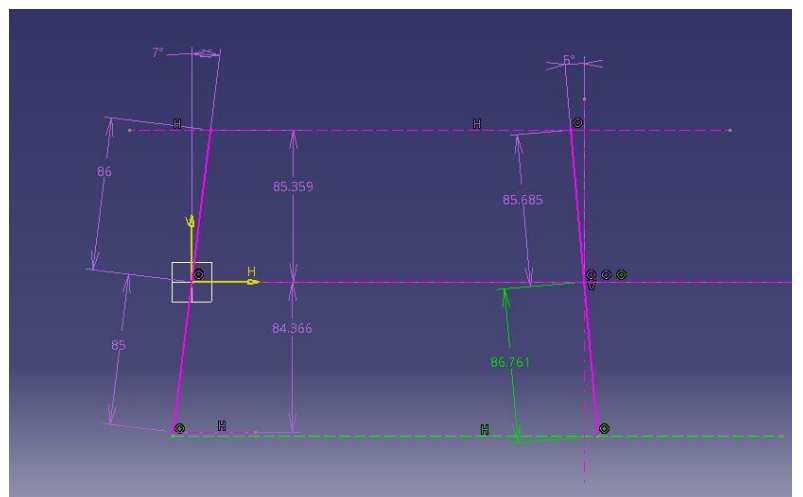

Fig.7 Effect of King Pin and Caster on Length 


\subsection{Design of Spindle}

Firstly the spindle is designed on which other components such as knuckle, bearings and hub will be fitted. At this stage we cannot decide the actual length of the spindle, so we just consider the tentative length of the spindle.

Material: - The material for manufacturing the spindle is taken to be EN24. There will be parts which will be press fitted on the spindle. So heat treatment will be necessary to increase surface hardness. Besides the yield strength in tension of EN24 is also high. Syt $=654 \mathrm{~N} / \mathrm{mm}^{2}[4]$

Endurance Limit $=412 \mathrm{~N} / \mathrm{mm}^{2}[4]$

It consists of following steps: -

\subsubsection{Determining the forces acting on the spindle:}

The forces acting on the spindle are as follows

a. Weight of the vehicle

During static and dynamic conditions a constant force of the self-weight is acting on the spindle at the part inside the knuckle. Even if it is considered as the more than half the weight of the car is acting at the front portion of the car during braking, the weight on the one wheel is

Weight in the front portion $=175 \mathrm{~kg}$

Weight on one tire $=175 / 2=87.5 \mathrm{~kg}$

Force due to weight of the vehicle $=87.5 \times 9.81=$ $858.375 \mathrm{~N}$

Let us consider this weight to be $\mathbf{1 0 0 0} \mathbf{N}$.

b. Bump force on the tire

At the time of a bump in the surface a force will act on the portion of the spindle which is inside the spindle. This is because the hub is bolted directly to the wheel. This force is obtained from the wheel rate. For design purpose the wheel rate is kept as $45 \mathrm{~N} / \mathrm{mm}^{2}$. Also it is considered that there will be no bump more than $30 \mathrm{~mm}$ as the track is extremely flat.

Bump Force $=$ Wheel rate $\times$ Travel due to bump

$$
=45 \times 30=\mathbf{1 3 5 0} \mathbf{N}
$$

c. Torque on the spindle.

Torque $=$ mass on the spindle $\times \mathrm{g} \times$ radius of the wheel $\times$ coefficient of friction

$$
\begin{aligned}
& =75 \times 9.81 \times \frac{(502.8 \times 0.5)}{1000} \times 0.7 \\
& =129.44 \mathrm{~N}
\end{aligned}
$$

Torque on spindle $=130 \mathrm{~N}$

\subsubsection{Drawing the Free Body Diagram}

Drawing the Free Body Diagram (FBD) gives an idea about the forces acting on the component simultaneously.

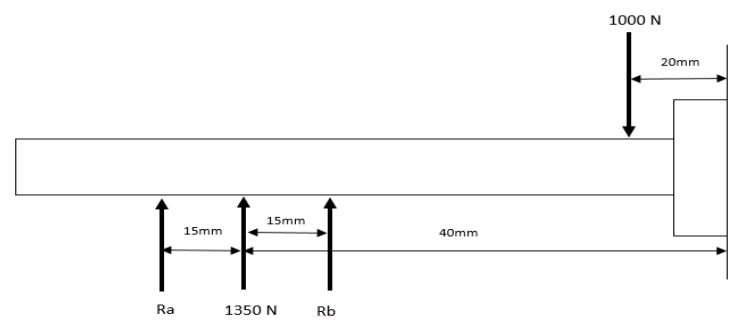

Fig.8 Free body Diagram

\subsubsection{Designing and determining the dimensions.}

\section{Shear Failure of the Spindle}

The spindle is likely to fail in shear because of the bump force. The spindle is a critical part and it is not at all desirable to fail any condition, hence the factor of safety is taken to be 3 .

The allowable shear stress $\tau=\frac{S y t \times 0.5}{F s}=\frac{654 \times 0.5}{3}=109$ $\mathrm{N} / \mathrm{mm}^{2}$

Now,

$$
\begin{gathered}
\tau=\frac{\text { Force }}{\text { Resisting Area }} \\
109=\frac{1350}{\frac{\pi}{4} d^{2}} \\
\therefore \boldsymbol{d}=\mathbf{3 . 9 7 1} \mathbf{~} \mathbf{m m}
\end{gathered}
$$

Thus from the shear failure the diameter of spindle-'d' comes out to be $4 \mathrm{~mm}$

Bending and Torsional Failure of the Spindle

To find out the Maximum Bending Moment, the SFD and BMD are to be drawn. Referring to the force diagram of the spindle, 

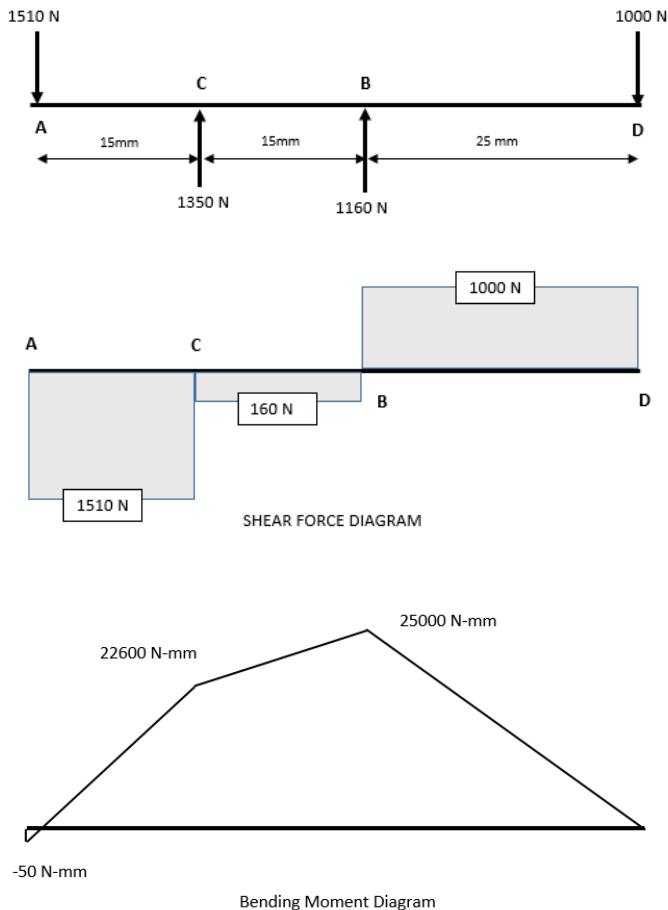

Fig.9 Shear Force Diagram and Bending Moment Diagram

Equating forces along Y-axis to 0

$\mathrm{Ra}+\mathrm{Rb}=1000-1350$

$\therefore \mathrm{Ra}+\mathrm{Rb}=-350 \mathrm{~N}$

Equating the moments about point $\mathrm{A}=0$

$\therefore-(1350 \times 15)-(\mathrm{Rb} \times 30)+(1000 \times 55)=0$

$\therefore \underline{\mathrm{Rb}}=1158.33 \mathrm{~N} \cong 1160 \mathrm{~N}$

$\therefore \mathrm{Ra}+1158.33 \mathrm{~N}=-350 \mathrm{~N}$

$\therefore \mathrm{Ra}=-1508.33 \mathrm{~N} \cong 1510 \mathrm{~N}$

After reconsidering the force diagram comes out be as shown in figure

Now,

Moment about $\mathrm{D}=0$

Moment about $\mathrm{B}=(1000 \times 25)=25000 \mathrm{~N}-\mathrm{mm}$

Moment about $C=(1000 \times 40)-(1160 \times 15)=22600$ $\mathrm{N}-\mathrm{mm}$

Moment about $A=(1000 \times 550)-(1160 \times 30)-(1350$ $\times 15)=-50 \mathrm{~N}-\mathrm{mm}$

From above it is clearly seen that the maximum bending moment is at point $\mathrm{B}$, thus $\mathrm{Mb}=25000 \mathrm{~N}-\mathrm{mm}$
The Torque acting on spindle can be directly taken from result number (i)

\section{$\therefore \mathrm{Mt}=130 \mathrm{~N}-\mathrm{m}=130000 \mathrm{~N}-\mathrm{mm}$}

As EN24 is a ductile material, using maximum shear stress theory to find out the diameter of the spindle.

$$
\begin{aligned}
& \mathrm{d}^{3}=\frac{16}{\pi \times \tau} \times \sqrt{\left(M b^{2}+M t^{2}\right)} \\
& \mathrm{d}^{3}=\frac{16}{\pi \times 109} \times \sqrt{\left(25000^{2}+130000^{2}\right)} \\
& \therefore \mathbf{d}=\mathbf{1 8 . 3 5} \mathbf{~ m m} \approx \mathbf{2 0} \mathbf{~ m m}
\end{aligned}
$$

\section{Thus from the bending and torsional failure the diameter of spindle-'d' comes out to be $20 \mathrm{~mm}$}

\subsubsection{Analysis and Optimization.}

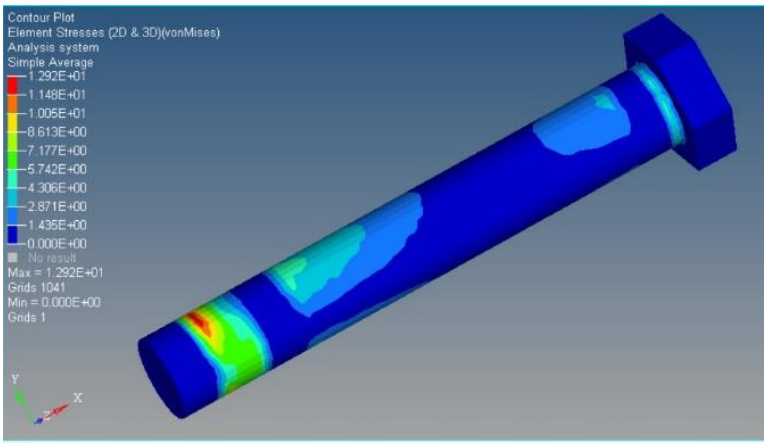

Fig.10 FEM Analysis of Spindle

\subsection{Design of Knuckle}

Knuckle is that part of the wheel assembly which is press fitted on the spindle and the A-arms are also mounted on the Knuckle. Besides the knuckle also serves the function of providing mounting to the Brake Caliper. The Steering Arm which is used to connect the wheel assembly and the tie rod is also mounted on the knuckle. Thus due to all these mountings, there are a lot of forces acting on the knuckle. The Knuckle as such is subjected to completely reversed types of stress while turning from one turn to the other and also during braking and accelerating. Thus a brittle material is not at suitable for this application. Thus taking a tensile material called Aluminum 7075 T6. It has high strength to weight ratio. Thus with much lower weight one can produce strong knuckles. The Endurance limit of this material is much more than that of other aluminum series. The material properties are as follows:-

$$
\text { Syt }=503 \mathrm{~N} / \mathrm{mm}^{2}[3]
$$

Endurance Limit $=160 \mathrm{~N} / \mathrm{mm}^{2}[3]$ 
Density $=2800 \mathrm{~kg} / \mathrm{m}^{3}$

\subsubsection{Determining the forces acting on the Knuckle:}

The forces acting on the spindle are as follows

a. Longitudinal Forces during Braking:-

While Braking, the weight of the rear side tends to come in the front side of the vehicle so there is a load transfer that is taking place form rear to front. It intern affects the knuckle as these forces act on the A-arm mounting points through the A-arms.

Considering Maximum acceleration of $1 \mathrm{~g}=9.81 \mathrm{~m} / \mathrm{s}^{2}$

Force at the front side $=$ mass at the rear side of the vehicle $\times$ acceleration

Let the mass at the rear side of the vehicle be 0.6 times the total weight

$\therefore$ Mass at the rear side of the vehicle $=0.6 \times 300=180$ $\mathrm{kg}$

Force $=180 \times 9.81$

$\therefore$ Force $=1765.8 \mathrm{~N}$

Now force on 1 wheel $=1765.8 / 2=882.9 \mathrm{~N}$

\section{Thus Longitudinal Force $=882.9 \mathrm{~N}$}

b. Lateral Forces: -

Lateral forces are because of two reasons - centrifugal force and lateral load transfer from outside to inside while turning. The centrifugal force is considered as follows

Let the vehicle take a turn of $6 \mathrm{~m}$ turning radius and at a speed of $30 \mathrm{kmph}$

$\mathrm{r}=$ turning radius $=6 \mathrm{~m}$

$\mathrm{V}=30 \mathrm{kmph}=\frac{30 \times 5}{18}=8.3333 \mathrm{~m} / \mathrm{s}$

Centrifugal Force $=\frac{m \times v^{2}}{r}=\frac{0.4 \times 300 \times 8.3333^{2}}{6}=\mathbf{1 3 8 8 . 7 7} \mathbf{N}$

Now consider if all the weigh at the front side comes on the wheel assembly the force will be Force due to lateral load transfer $=0.4 \times 300 \times 9.81=\mathbf{1 1 7 5 . 5}$

c. Force on the Steering Arm: -

According to the steering effort, the force on steering arm was found out to be 1165.52 at an angle of $8.556^{\circ}$. After resolving these forces.
Force $1=1165.82 \times \cos (8.556)=1152.84 \mathrm{~N}$

Force $2=1165.82 \times \sin (8.556)=173.44 \mathrm{~N}$

But the force on steering arm $=1165.52 \mathrm{~N}$

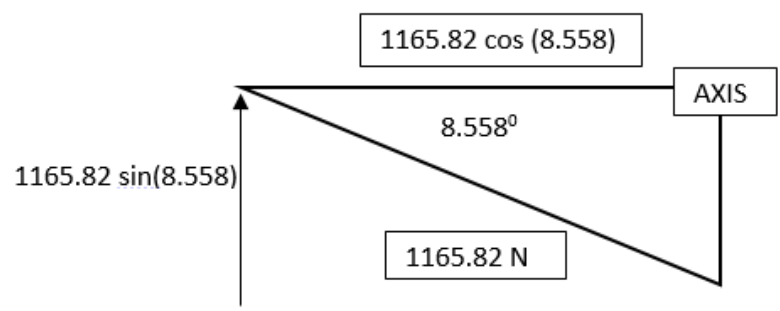

Fig.11 Steering Arm Forces

d. Forces on the caliper mounting points due to torque

The radius for the upper and lower caliper mount points are $89.13 \mathrm{~mm}$ and $60.233 \mathrm{~mm}$. The maximum force will be at minimum radius. Hence consider the force on the lower arm.

Force (lower) $=\frac{\text { Torque }}{\text { radius }}=\frac{130000}{60.233}=2158.28 \mathrm{~N}$

\subsubsection{Drawing the Free Body Diagram}

Drawing the Free Body Diagram (FBD) gives an idea about the forces acting on the component simultaneously.

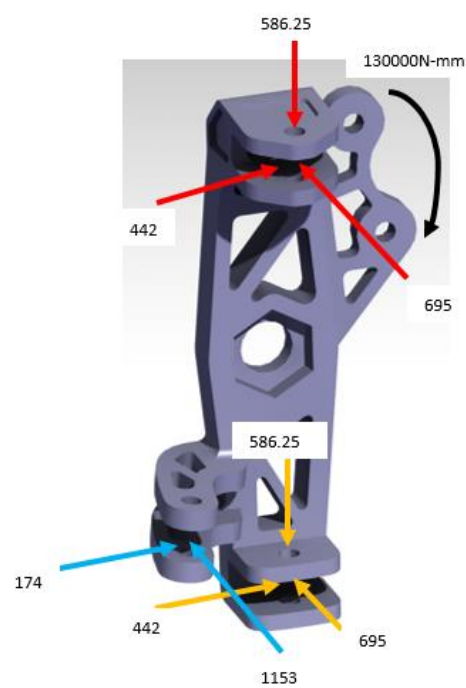

Fig.12 Free Body Diagram

3.4.3 Designing and determining the dimensions.

Selection of Knuckle Upper and Lower Bracket Bolt 
The bolts are standard parts and have a defined value of yield strength. All bolts used in the Wheel Assembly are made up of a minimum of 8.8 Grade.

Syt $=580 \mathrm{~N} / \mathrm{mm}^{2}[3]$

Factor of Safety $=2$

Shear Force acting on these bolts $=\sqrt{442^{2}+695^{2}}=$ $823.64 \mathrm{~N}$

Shear Stress on the bolts $=\tau=\frac{\text { Syt } \times 0.5}{\text { Factor of Safety }}=\frac{580 \times 0.5}{2}$ $=145 \mathrm{~N} / \mathrm{mm}^{2}$

Now, $\tau=\frac{\text { Force }}{\text { Area }}$

$\therefore 145=\frac{823.64}{2 \times\left(\frac{\pi}{4} d_{c}^{2}\right)}$

$\therefore d_{c}=1.9016 \mathrm{~mm}$

$\therefore d=d_{c} / 0.8$

$\therefore d=1.9016 / 0.8$

$\therefore d=2.37 \mathrm{~mm}$

This value is too small. Thus for practical reasons selecting the bracket bolt size as M8.

\section{Selection of Caliper Mounting Bolt}

Shear Stress on the bolts $=\tau=\frac{\text { Syt } \times 0.5}{\text { Factor of Safety }}=\frac{580 \times 0.5}{2}$ $=145 \mathrm{~N} / \mathrm{mm}^{2}$

The force acting on the caliper bolt is given by the result (vi)

$$
\begin{aligned}
& \text { Now, } \tau=\frac{\text { Force }}{\text { Area }} \\
& \therefore 145=\frac{2158.23}{\left(\frac{\pi}{4} d_{c}^{2}\right)} \\
& \therefore d_{c}=4.3533 \mathrm{~mm} \\
& \therefore d=d_{c} / 0.8 \\
& \therefore d=4.3533 / 0.8 \\
& \therefore d=5.44 \mathrm{~mm}
\end{aligned}
$$

Thus selecting the caliper bolt size as M8.

\section{Selection of Steering arm Bolt}

Shear Stress on the bolts $=\tau=\frac{\text { Syt } \times 0.5}{\text { Factor of Safety }}=\frac{580 \times 0.5}{2}$ $=145 \mathrm{~N} / \mathrm{mm}^{2}$
The force acting on the Steering arm is given by the result (v)

Now, $\tau=\frac{\text { Force }}{\text { Area }}$

$\therefore 145=\frac{1165.52}{2 \times\left(\frac{\pi}{4} d_{c}^{2}\right)}$

$\therefore d_{c}=2.2621 \mathrm{~mm}$

$\therefore d=d_{c} / 0.8$

$\therefore d=2.2621 / 0.8$

$\therefore d=2.82 \mathrm{~mm}$

Thus selecting the Steering arm bolt size as M8.

Longitudinal Shear Failure of the Knuckle Bracket.

Allowable stress in the knuckle in shear $=\tau=$ $\frac{\text { Syt } \times 0.5}{\text { Factor of Safety }}=\frac{503 \times 0.5}{2}=125.75 \mathrm{~N} / \mathrm{mm}^{2}$

Longitudinal force acting on bracket $=442 \mathrm{~N}$

Now, $\tau=\frac{\text { Force }}{\text { Area }}$

$\therefore 125.75=\frac{442}{2 \times(t \times b)}$

$\mathrm{t} \times \mathrm{b}=1.7574$

Where,

$\mathrm{t}=$ thickness of one plate of bracket

$\mathrm{b}=$ distance between the hole and the end of bracket

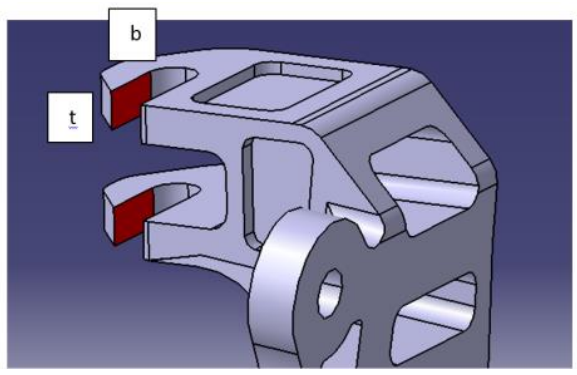

Fig.13 Shear of Knuckle Bracket If $\mathrm{t}=6 \mathrm{~mm} \mathrm{~b}=0.3 \mathrm{~mm}$

Thus for practical reasons the width of bracket in longitudinal direction is taken to be $12 \mathbf{~ m m}$

The thickness of the bracket is taken as $6 \mathbf{m m}$

Lateral Shear Failure of the Knuckle Bracket 
Allowable stress in the knuckle in shear $=\tau=$ $\frac{\text { Syt } \times 0.5}{\text { Factor of Safety }}=\frac{503 \times 0.5}{2}=125.75 \mathrm{~N} / \mathrm{mm}^{2}$

Lateral force acting on bracket $=695 \mathrm{~N}$

$$
\text { Now, } \tau=\frac{\text { Force }}{\text { Area }}
$$

$\therefore 125.75=\frac{695}{2 \times(t \times b)}$

$\mathrm{t} \times \mathrm{b}=2.7634$

Where,

$\mathrm{t}=$ thickness of one plate of bracket

$b=$ distance between the hole and the end of bracket

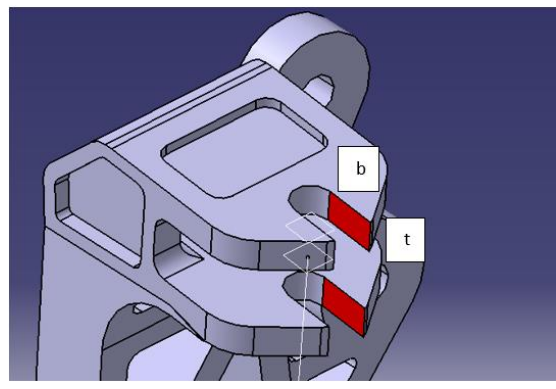

Fig.14 Shear of Knuckle Bracket

If $\mathrm{t}=6 \mathrm{~mm} \mathrm{~b}=0.46 \mathrm{~mm}$

Thus for practical reasons the width of bracket in lateral direction is taken to be $\mathbf{1 0} \mathbf{~ m m}$

\section{The thickness of the bracket is taken as $6 \mathbf{~ m m}$}

\section{Bending Failure of Knuckle}

\section{Bending due to Longitudinal Force}

This bending is due to the force of $883 \mathrm{~N}$. The longest part of knuckle is $86 \mathrm{~mm}$ away from the center and the knuckle is almost symmetric. Thus bending moment in such cases is taken to be

$\mathrm{Mb}=883 \times 0.5 \times 86$

$\mathrm{Mb}=37969 \mathrm{~N}-\mathrm{mm}$

Now,

By Flexural Equation,

$$
\begin{gathered}
\frac{M_{b}}{I}=\frac{\sigma_{b}}{y} \\
\sigma_{b}=\frac{\text { Syt }}{\text { Factor of Safety }}=\frac{503}{2}=251.5 \mathrm{~N} / \mathrm{mm}^{2}
\end{gathered}
$$

$$
\begin{aligned}
& \mathrm{y}=\mathrm{b} / 2 \\
& \mathrm{I}=\frac{1}{12} t b^{3}
\end{aligned}
$$

Where,

$\mathrm{t}=$ thickness of knuckle

$\mathrm{b}=$ width of knuckle

$$
\begin{aligned}
& \frac{37969}{\frac{1}{12} t b^{3}}=\frac{251.5}{\mathrm{~b} / 2} \\
& \mathrm{t} \times \mathrm{b}^{3}=905.821
\end{aligned}
$$

If $\mathrm{t}=16 \mathrm{~mm} \mathrm{~b}=3.84 \mathrm{~mm}$

But here it is also important to understand that the spindle will be fitting in the knuckle thus for this reason the width of knuckle is taken as $50 \mathrm{~mm}$ at the center and would then decrease to $40 \mathrm{~mm}$ till the end.

\section{Thus, the width of knuckle is $50 \mathrm{~mm}$.}

\section{The thickness of knuckle is 16 mm.}

\section{Bending due to Centrifugal Force}

This bending is due to the force of $1388.77 \mathrm{~N}$. The longest part of knuckle is $86 \mathrm{~mm}$ away from the center and the knuckle is almost symmetric. Thus bending moment in such cases is taken to be

$\mathrm{Mb}=1388.77 \times 0.5 \times 86$

$\mathrm{Mb}=59717.11 \mathrm{~N}-\mathrm{mm}$

Now,

By Flexural Equation,

$$
\begin{aligned}
& \qquad \frac{M_{b}}{I}=\frac{\sigma_{b}}{y} \\
& \sigma_{b}=\frac{\text { Syt }}{\text { Factor of Safety }}=\frac{503}{2}=251.5 \mathrm{~N} / \mathrm{mm}^{2} \\
& \mathrm{y}=\mathrm{t} / 2 \\
& \mathrm{I}=\frac{1}{12} b t^{3}
\end{aligned}
$$

Where,

$\mathrm{t}=$ thickness of knuckle

$\mathrm{b}=$ width of knuckle

$$
\frac{59717.11}{\frac{1}{12} b t^{3}}=\frac{251.5}{t / 2}
$$

$\mathrm{b} \times \mathrm{t}^{3}=1424.66$ 
If $\mathrm{t}=16 \mathrm{~mm} \mathrm{~b}=0.3478 \mathrm{~mm}$

But here it is also important to understand that the spindle will be fitting in the knuckle thus for this reason the width of knuckle is taken as $50 \mathrm{~mm}$ at the center and would then decrease to $40 \mathrm{~mm}$ till the end.

Thus, the width of knuckle is $50 \mathrm{~mm}$.

The thickness of knuckle is $\mathbf{1 6} \mathbf{~ m m}$.

\section{Shear Failure of the Steering Arm}

Allowable stress in the knuckle in shear $=\tau=$ $\frac{\text { Syt } \times 0.5}{\text { Factor of Safety }}=\frac{503 \times 0.5}{2}=125.75 \mathrm{~N} / \mathrm{mm}^{2}$

Force acting on Steering Arm as obtained from result (v) is $1165 \mathrm{~N}$

Now, $\tau=\frac{\text { Force }}{\text { Area }}$

$\therefore 125.75=\frac{1165}{2 \times(t \times b)}$

$t \times b=4.6322$

Where,

$\mathrm{t}=$ thickness of one plate of steering arm

$\mathrm{b}=$ distance between the hole and the end of arm

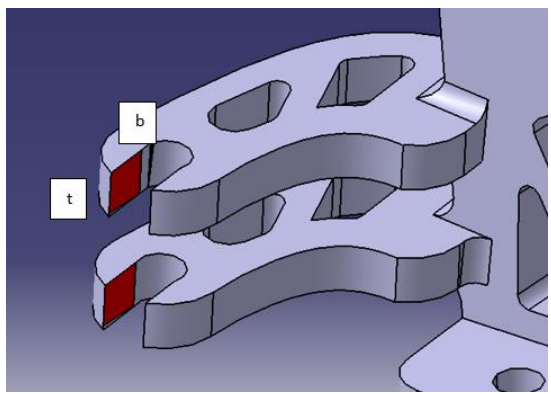

Fig.15 Shear of Steering Arm

If $\mathrm{t}=6 \mathrm{~mm} \mathrm{~b}=0.77 \mathrm{~mm}$

Thus for practical reasons the width of steering arm is taken to be $6 \mathbf{~ m m}$

\section{The thickness of the bracket is taken as $6 \mathrm{~mm}$}

\section{Bending Failure of Steering Arm}

This bending is due to the force of $1165 \mathrm{~N}$. The longest part of steering arm is $50 \mathrm{~mm}$ away from the center and the knuckle Thus bending moment in such cases is taken to be

$\mathrm{Mb}=1165 \times 50$

$\mathrm{Mb}=58250 \mathrm{~N}-\mathrm{mm}$

Now,

By Flexural Equation,

$$
\frac{M_{b}}{I}=\frac{\sigma_{b}}{y}
$$

$$
\begin{aligned}
& \sigma_{b}=\frac{\text { Syt }}{\text { Factor of Safety }}=\frac{503}{2}=251.5 \mathrm{~N} / \mathrm{mm}^{2} \\
& \mathrm{y}=\mathrm{t} / 2 \\
& \mathrm{I}=\frac{1}{12} b t^{3}
\end{aligned}
$$

Where,

$\mathrm{t}=$ thickness of steering arm in contact with knuckle $b=$ width of steering arm in contact with knuckle

$$
\frac{58250}{\frac{1}{12} b t^{3}}=\frac{251.5}{t / 2}
$$

$$
t \times b^{3}=1389.66
$$

If $\mathrm{t}=8 \mathrm{~mm} \mathrm{~b}=5.579 \mathrm{~mm}$

Thus, the width of steering arm is $6 \mathbf{m m}$.

The thickness of steering arm is $8 \mathbf{~ m m}$.

\section{Shear Failure of the Caliper Mounting}

Allowable stress in the knuckle in shear $=\tau=$ $\frac{\text { Syt } \times 0.5}{\text { Factor of Safety }}=\frac{503 \times 0.5}{2}=125.75 \mathrm{~N} / \mathrm{mm}^{2}$

Force acting on Steering Arm as obtained from result (vi) is $2158.28 \mathrm{~N}$

Now, $\tau=\frac{\text { Force }}{\text { Area }}$

$\therefore 125.75=\frac{2158.28}{(t \times b)}$

$\mathrm{t} \times \mathrm{b}=17.163$

Where,

$\mathrm{t}=$ thickness of caliper mount

$b=$ distance between the hole and the end of mount 


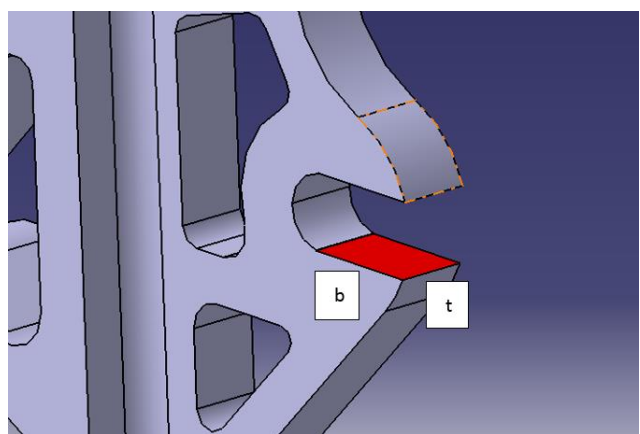

Fig.16 Shear of Caliper Mount

If $\mathrm{t}=6 \mathrm{~mm} \mathrm{~b}=2.86 \mathrm{~mm}$

Thus the width of the caliper mount is taken to be 8 mm

\section{The thickness of the bracket is taken as $6 \mathrm{~mm}$.}

On the basis of the dimensions found from above calculations, CAD- Part of the knuckle is drawn. This is ten used to carry out analysis of the component. Care should be taken while deciding the dimensions on the CAD-Part. The dimensions of the component obtained from the calculations are the minimum dimensions so that the stress values in that component does not exceed a particular value, thus the actual dimensions must be equal to or greater than the calculated dimensions.

\subsubsection{Analysis and Optimization.}

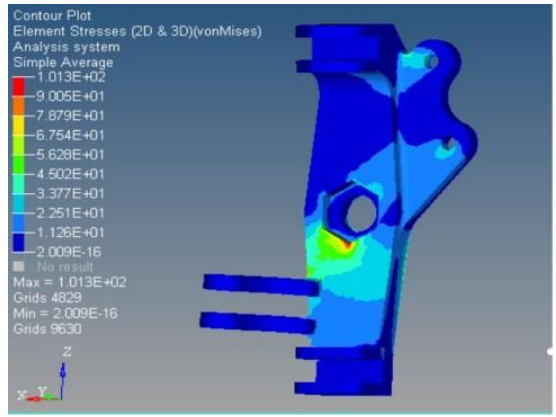

Fig.17 Analysis 1

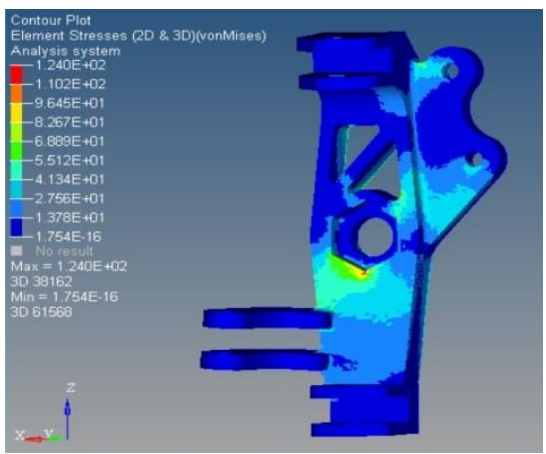

Fig.18 Analysis 2

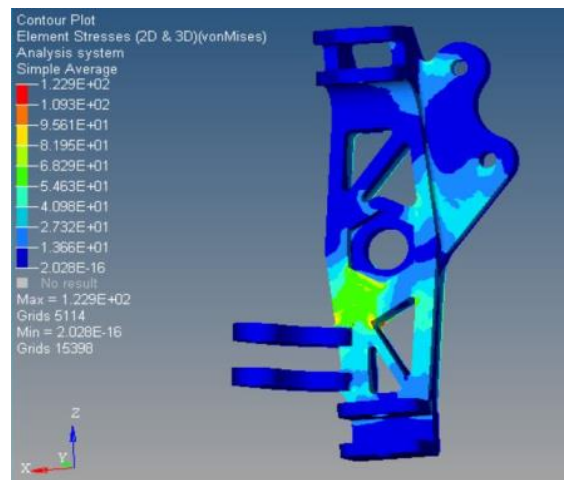

Fig.19 Analysis 3

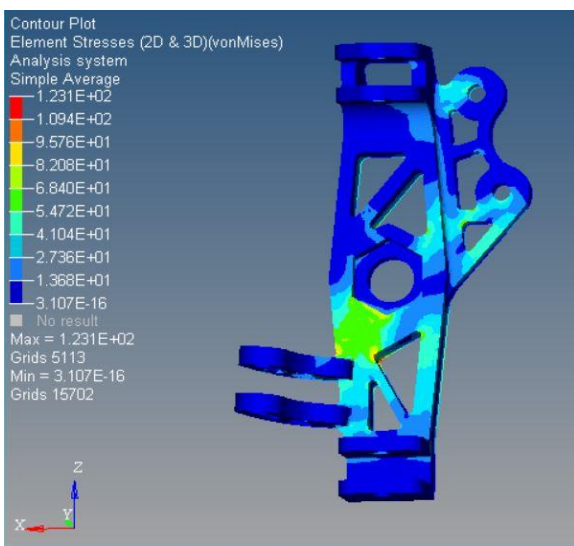

Fig.20 Analysis 4

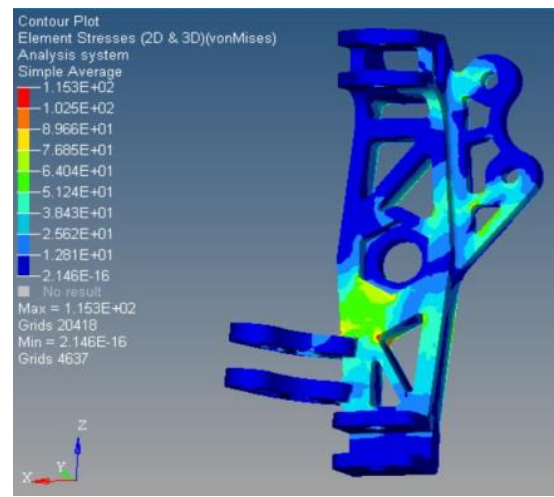

Fig.21 Analysis 5

From the above analysis results, it was concluded that material must be reduced from the less stress concentration areas. Thus by step by step reeducation in material it was made sure that the design was of low weight with stresses under the permissible limit, thus creating an optimized Design.

\subsection{Selection of bearing}

At the front wheels, there are axial as well as radial forces which are acting on the bearing. This is due to the reason that the front wheel also has to steer the vehicle and also due to centrifugal forces while 
cornering. Thus it becomes important to used taper roller bearing which can sustain radial as well as axial forces. Furthermore one must use 2 taper roller bearings in order to cancel out the forces. Otherwise there would be unbalancing of the forces.

This is not the case in Rear Wheels. In rear wheels there are centrifugal forces acting, but the wheels are subjected to high speeds and torques. Taper roller bearing is incapable of sustaining such high speeds and torques. Deep groove ball bearing can sustain such high speeds and torques and hence at rear side deep groove ball bearing has been used. Besides, it is not at all necessary to used 2 deep groove ball bearings, one is also sufficient.

The type of arrangement used is back-to-back. The radial forces on the bearings are $700 \mathrm{~N}$ and $634 \mathrm{~N}$. Also the radial force acting on the bearing is $1388 \mathrm{~N}$. According to the calculations done with reference to the manufacturer's catalog [2], it was found that the bearing 32004X_Q is suitable for the front wheel assembly, as its value of $\mathrm{C}$ is less than $22900 \mathrm{~N}$.

The Specifications of the bearing ${ }^{[2]}$ are

Inner diameter of bearing $=20 \mathrm{~mm}$

Outer diameter of bearing $=42 \mathrm{~mm}$

Thickness of bearing $=15 \mathrm{~mm}$

\subsection{Design of Hub}

Hub is the part of wheel assembly on which the wheel and disk are mounted. Both the Wheel as well as the disk are mounted on the hub with the help of bolts. As discussed earlier the outer race of the bearing is press fitted in the hub and hence provision is made in the hub to enclose the bearing. The Hub itself is made of 2 Petal parts. One of the wheel and the other of the brake disk

\subsubsection{Determining the forces acting on the Hub: -}

The following Forces are acting on the Hub.

\section{a. Torque on the Brake Disk Petal : -}

A torque of $130 \mathrm{Nm}$ is acting on the Brake Disk Petal.

The Force acting on each hole $=\frac{\text { Moment } \div \text { Radis }}{\text { No of holes }}=$ $\frac{130000 \div 100 / 2}{3}=880 \mathrm{~N}$

\section{b. Torque on the Wheel Petal : -}

In order to sustain this braking effect the wheel must also provide and equal and opposite torque. Thus the magnitude of torque is same but the direction is opposite.
The Force acting on each hole $=\frac{\text { Moment } \div \text { Radis }}{\text { No of holes }}=$ $\frac{130000 \div 98.5 / 2}{3}=879.86 \mathrm{~N}$

\section{c. Force due to Side Impact: -}

If the vehicle is banged by other vehicle from side or if the vehicle has a collision with the fencing from side, there are chances that the petals might bend. Hence this side impact force must also be considered.

Here the side Impact force is taken to be $2 \mathrm{G}=2 \times \mathrm{g} \times$ vehicle mass

$\therefore$ Impact force $=2 \times 9.81 \times 300=5886 \mathrm{~N}$

$\therefore$ Impact force on 1 petal $=8829 / 3=1962 \mathrm{~N}$

\section{d. Loads on Bearing: -}

The load on 1 bearing is $700 \mathrm{~N}$

The load on $2^{\text {nd }}$ bearing is $634 \mathrm{~N}$

The axial load on the bearing is $1388 \mathrm{~N}$

\subsubsection{Drawing the Free Body Diagram}

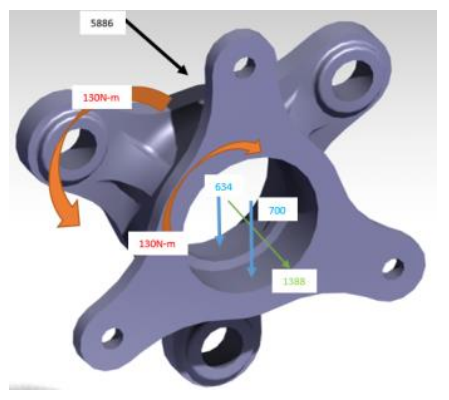

Fig.22 Free Body Diagram

\subsubsection{Designing and determining the dimensions}

\section{Selection of Wheel Bolt}

Shear Stress on the bolts $=\tau=\frac{\text { Syt } \times 0.5}{\text { Factor of Safety }}=\frac{580 \times 0.5}{2}$ $=145 \mathrm{~N} / \mathrm{mm}^{2}$

The force acting on the Wheel Bolt is given by the result (vii)

$$
\begin{aligned}
& \text { Now, } \tau=\frac{\text { Force }}{\text { Area }} \\
& \therefore 145=\frac{880}{\left(\frac{\pi}{4} d_{c}^{2}\right)}
\end{aligned}
$$


$\therefore d_{c}=2.99 \mathrm{~mm}$

$\therefore d=d_{c} / 0.8$

$\therefore d=2.99 / 0.8$

$\therefore d=3.74 \mathrm{~mm}$

Also in the rim there is a provision of M12 Bolt. Hence selecting bolt of M12.

\section{Selection of Brake Disk Bolt}

Shear Stress on the bolts $=\tau=\frac{\text { Syt } \times 0.5}{\text { Factor of Safety }}=\frac{275 \times 0.5}{2}$ $=68.75 \mathrm{~N} / \mathrm{mm}^{2}$

The force acting on the Wheel Bolt is given by the result (viii)

$$
\begin{aligned}
& \text { Now, } \tau=\frac{\text { Force }}{\text { Area }} \\
& \therefore 68.75=\frac{879.89}{\left(\frac{\pi}{4} d_{c}^{2}\right)} \\
& \therefore d_{c}=2.99 \mathrm{~mm} \\
& \therefore d=d_{c} / 0.8 \\
& \therefore d=2.99 / 0.8 \\
& \therefore d=3.74 \mathrm{~mm}
\end{aligned}
$$

Thus selecting the bolt size of $\mathbf{M 8}$.

\section{Design of Wheel Petal}

\section{Shear Failure of Petal}

Allowable stress in the Hub in shear $=\tau=$ $\frac{\text { Syt } \times 0.5}{\text { Factor of Safety }}=\frac{503 \times 0.5}{2}=125.75 \mathrm{~N} / \mathrm{mm}^{2}$

Force acting on Petal as obtained from result (vii) is $880 \mathrm{~N}$

Now, $\tau=\frac{\text { Force }}{\text { Area }}$

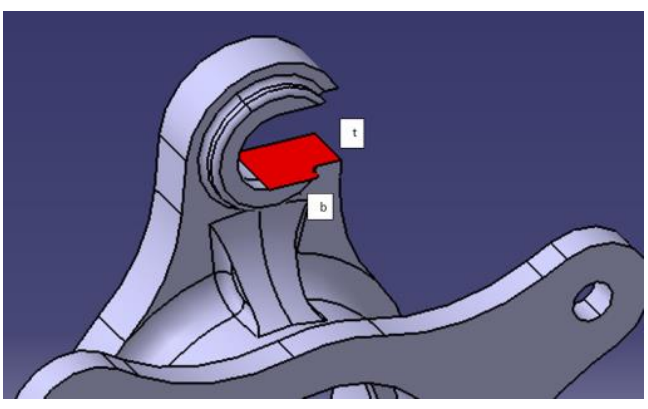

Fig.23 Shear of Wheel Petal $\therefore 125.75=\frac{880}{2 \times(t \times b)}$

$\mathrm{t} \times \mathrm{b}=3.5$

Where,

$\mathrm{t}=$ thickness of Wheel Petal

$\mathrm{b}=$ distance between the hole and the end of petal

If $\mathrm{t}=8 \mathrm{~mm} \mathrm{~b}=0.475 \mathrm{~mm}$

Thus the width of the petal is taken to be $7 \mathbf{m m}$

The thickness of the petal is taken as $8 \mathrm{~mm}$.

\section{Bending of Wheel Petal}

This bending is due to the force of $880 \mathrm{~N}$. The radius of effective bending is $49.25 \mathrm{~mm}$

$\mathrm{Mb}=880 \times 49.25$

$\mathrm{Mb}=43340 \mathrm{~N}-\mathrm{mm}$

Now,

By Flexural Equation,

$$
\begin{aligned}
& \qquad \frac{M_{b}}{I}=\frac{\sigma_{b}}{y} \\
& \sigma_{b}=\frac{\text { Syt }}{\text { Factor of Safety }}=\frac{503}{2}=251.5 \mathrm{~N} / \mathrm{mm}^{2} \\
& \mathrm{y}=2 \mathrm{~b} / 2 \\
& 2 \mathrm{~b}=\mathrm{d} \\
& \mathrm{I}=\frac{1}{12} t d^{3}
\end{aligned}
$$

Where,

$\mathrm{t}=$ thickness of knuckle

$b=$ width of knuckle

$$
\frac{43340}{\frac{1}{12} t d^{3}}=\frac{251.5}{\mathrm{~d} / 2}
$$

$t \times d^{3}=1034$

If $\mathrm{t}=8 \mathrm{~mm} \mathrm{~d}=11.36 \mathrm{~mm}$

Thus, the width of knuckle is $\mathbf{1 2} \mathbf{m m}$.

The thickness of knuckle is $8 \mathbf{~ m m}$.

Total thickness is $=$ width + diameter of hole $=12+14=26 \mathrm{~mm}$

\section{Design of Brake Disk Petal}

\section{Shear Failure of Petal}

Allowable stress in the Hub in shear $=\tau=$ $\frac{\text { Syt } \times 0.5}{\text { Factor of Safety }}=\frac{503 \times 0.5}{2}=125.75 \mathrm{~N} / \mathrm{mm}^{2}$ 
$\frac{M_{b}}{I}=\frac{\sigma_{b}}{y}$

$\sigma_{b}=\frac{\text { syt }}{\text { Factor of Safety }}=\frac{503}{2}=251.5 \mathrm{~N} / \mathrm{mm}^{2}$

$\mathrm{y}=\mathrm{t} / 2$

$\mathrm{I}=\frac{1}{12} b t^{3}$

Where,

$\mathrm{t}=$ thickness of petal

$\mathrm{b}=$ width of petal $=27 \mathrm{~mm}$ as found from above calculation

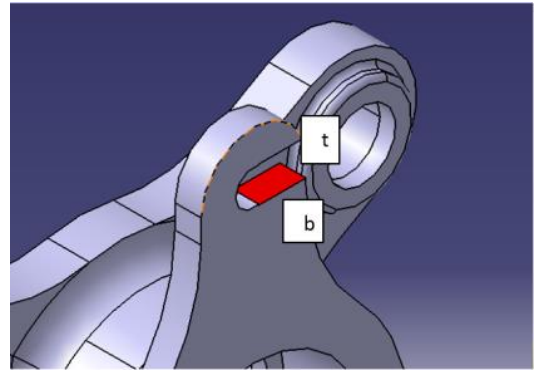

Fig.25 Hub Analysis 1

Force acting on Petal as obtained from result (vii) is $880 \mathrm{~N}$

Now, $\tau=\frac{\text { Force }}{\text { Area }}$

$$
\begin{aligned}
& \frac{49540.5}{\frac{1}{12} 27 t^{3}}=\frac{251.5}{t / 2} \\
& t=6.61 \mathrm{~mm}
\end{aligned}
$$

The thickness of petal is taken as $8 \mathbf{~ m m}$.

\subsubsection{Analysis and Optimization}

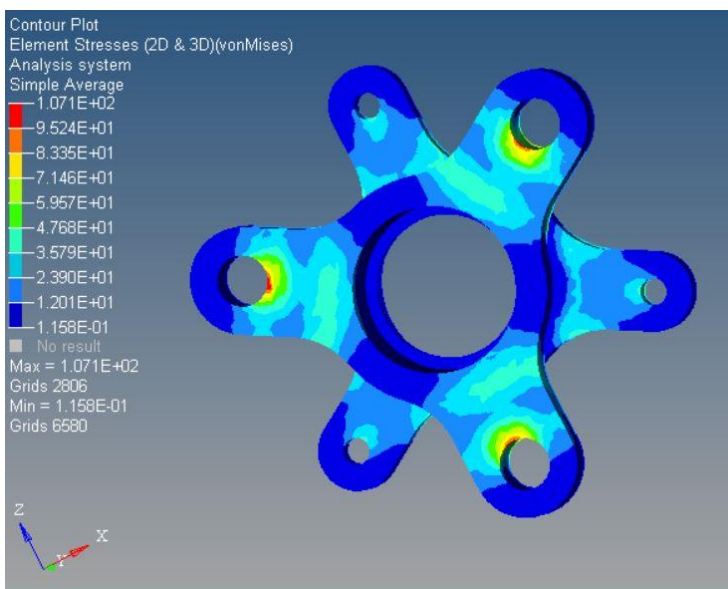

$\therefore 125.75=\frac{879.89}{2 \times(t \times b)}$

$t \times b=1.7495$

Where,

$\mathrm{t}=$ thickness of Wheel Petal

$b=$ distance between the hole and the end of petal

If $\mathrm{t}=5 \mathrm{~mm} \mathrm{~b}=0.35 \mathrm{~mm}$

Thus the width of the petal is taken to be $5 \mathrm{~mm}$.

\section{The thickness of the petal is taken as $6 \mathrm{~mm}$.}

\section{Design of Wheel petal due to Side Impact}

The force is obtained from the result (ix) for 1 petal $=$ $1962 \mathrm{~N}$

The effective bending Radius $=25.25 \mathrm{~mm}$

$\mathrm{Mb}=1962 \times 25.25$

$\mathrm{Mb}=49540.5 \mathrm{~N}-\mathrm{mm}$

Now,

By Flexural Equation,

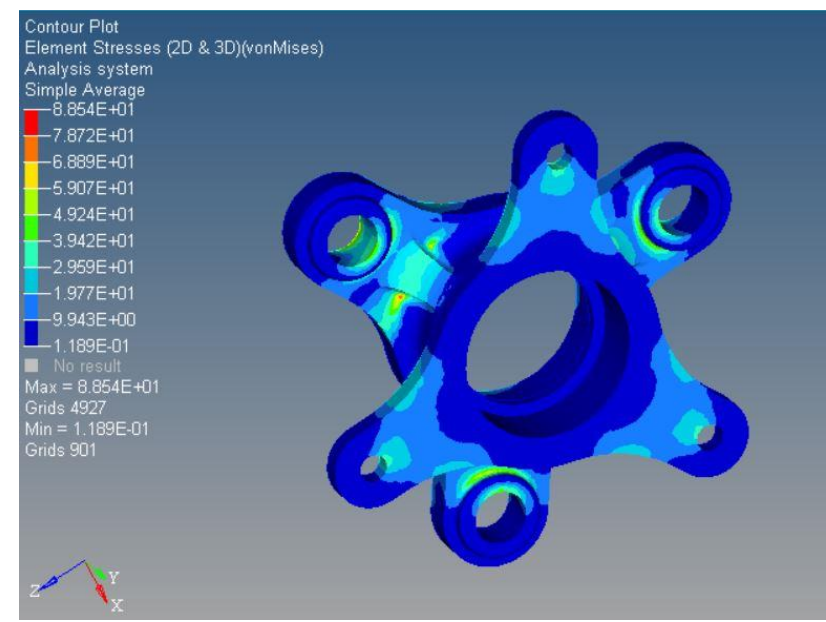

Fig.26 Hub Analysis 2

From the above result it is clear that hub without fillet and triangulation support were more prone to failure than those with fillet and triangulation support. 
Besides the stress concentration in the optimized design was less.

Table 3 Checking Parameters

\begin{tabular}{|c|c|c|c|}
\hline Part & $\begin{array}{c}\text { Stress } \\
\text { Induced }\end{array}$ & $\begin{array}{c}\text { Endurance } \\
\text { Strength }\end{array}$ & $\begin{array}{c}\text { Factor of } \\
\text { Safety }\end{array}$ \\
\hline Knuckle & 115 & 160 & 1.4 \\
\hline Hub & 88 & 160 & 1.8 \\
\hline Spindle & 13 & 392 & 30 \\
\hline
\end{tabular}

\section{Conclusions}

Following conclusions can be drawn from the paper,

1) For a component undergoing fatigue loading, the design criteria must always be Fatigue or Endurance Strength.

2) For carrying out optimization, material should be removed from the low stress concentration areas.

3) In order to minimize stress concentration areas, sharp corners and edges should be avoided.

4) If the component is subjected to fatigue failure like knuckle, then analysis of the components must be carried out in order to obtain actual stresses induced in the component.

5) For accurate results of analysis, mesh quality must be high and failing elements must be less than $3 \%$.

6) As spindle serves as a component on which the assembly is press fitted, its factor of safety is taken high.

\section{References}

P. Vishwakarma, M. Kanungoo, (2014), Finite Element Analysis Of Chervolet Front Hub With The Help Of Inventer, International Journal in IT and Engineering, vol.2, Issue 2, ISSN: 2321-1776.

G. Fisher,V. V. Graubisic, (1998), Design consideration and durability approval of wheel hub, SAE international,11-16-1998.

S.dhar, (1988), Fracturer analysis of wheel hub fabricated from pressure die aluminium assembly, Theoretical and applied fracturer mechanics, vol 0902-1988.

S. Li, Z. Lindu, (2009), Research on Optimization of Hub-And-Spoke Logistics Network Design with Impedance Effect, POMS 20th Annual Conference Orlando, Florida, U.S.A, 011-0370.

V.B.Bhandari, (1999), Design of Machine Elements, $8^{\text {th }}$ edition, Wiley.

Kutz M., (1986), Mechanical engineering handbook, New York, Wiley 